\title{
Correction to: Injectable hyaluronic acid-based antibacterial hydrogel adorned with biogenically synthesized AgNPs-decorated multi-walled carbon nanotubes
}

\author{
Pooyan Makvandi ${ }^{1}$ (D) Milad Ashrafizadeh ${ }^{2,3} \cdot$ Matineh Ghomi $^{4} \cdot$ Masoud Najafi, $^{5,6}$ Hamid Heydari Sheikh Hossein ${ }^{7}$. \\ Ali Zarrabi ${ }^{3}$ - Virgilio Mattoli ${ }^{1} \cdot$ Rajender S. Varma ${ }^{8}$
}

Published online: 6 November 2021

(c) The Author(s), under exclusive licence to Islamic Azad University 2021

\section{Correction to: Progress in Biomaterials (2021) 10:77-89 https://doi.org/10.1007/s40204-021-00155-6}

The authors found that the TEM image of AgNPs (Fig. 4a) is sent wrongly by the institute that took the TEM image. Even though the TEM images of pure AgNPs (in comparison with Ag-adorned CNTs) were not determinative and it does not affect the other results in this study, as responsibility of the authors to report precise and correct data, a new image (by TEM, Zeiss-EM10C-80 KV, Germany) was taken and now replaced in Fig. 4a. It is worth mentioning that other data and images in Fig. 4 are not altered. The authors do apologize for this inconvenience.

Publisher's Note Springer Nature remains neutral with regard to jurisdictional claims in published maps and institutional affiliations.
The original article can be found online at https://doi.org/10.1007/ s40204-021-00155-6.

\section{Pooyan Makvandi \\ Pooyan.makvandi@iit.it \\ $\triangle$ Matineh Ghomi \\ ma_gh@rocketmail.com \\ $\triangle$ Masoud Najafi najafi_ma@yahoo.com}

1 Istituto Italiano di Tecnologia, Centre for Materials Interface, viale Rinaldo Piaggio 34, 56025 Pontedera, Pisa, Italy

2 Department of Basic Science, Faculty of Veterinary Medicine, University of Tabriz, 51666-16471 Tabriz, Iran

3 Sabanci University Nanotechnology Research and Application Center (SUNUM), 34956 Tuzla, Istanbul, Turkey
4 Chemistry Department, Faculty of Science, Shahid Chamran University of Ahvaz, 61537-53843 Ahvaz, Iran

5 Medical Technology Research Center, Institute of Health Technology, Kermanshah University of Medical Sciences, 6715847141 Kermanshah, Iran

6 Radiology and Nuclear Medicine Department, School of Paramedical Sciences, Kermanshah University of Medical Sciences, Kermanshah, Iran

7 Department of Biotechnology, Faculty of Biological Science and Technology, University of Isfahan, Isfahan, Iran

8 Regional Centre of Advanced Technologies and Materials, Palacky University, Slechtitelu 27, 78371 Olomouc, Czech Republic 
Fig. 4 TEM images of $\mathbf{a}$ biosynthesized AgNPs and b MWCNTs decorated with biosynthesized AgNPs
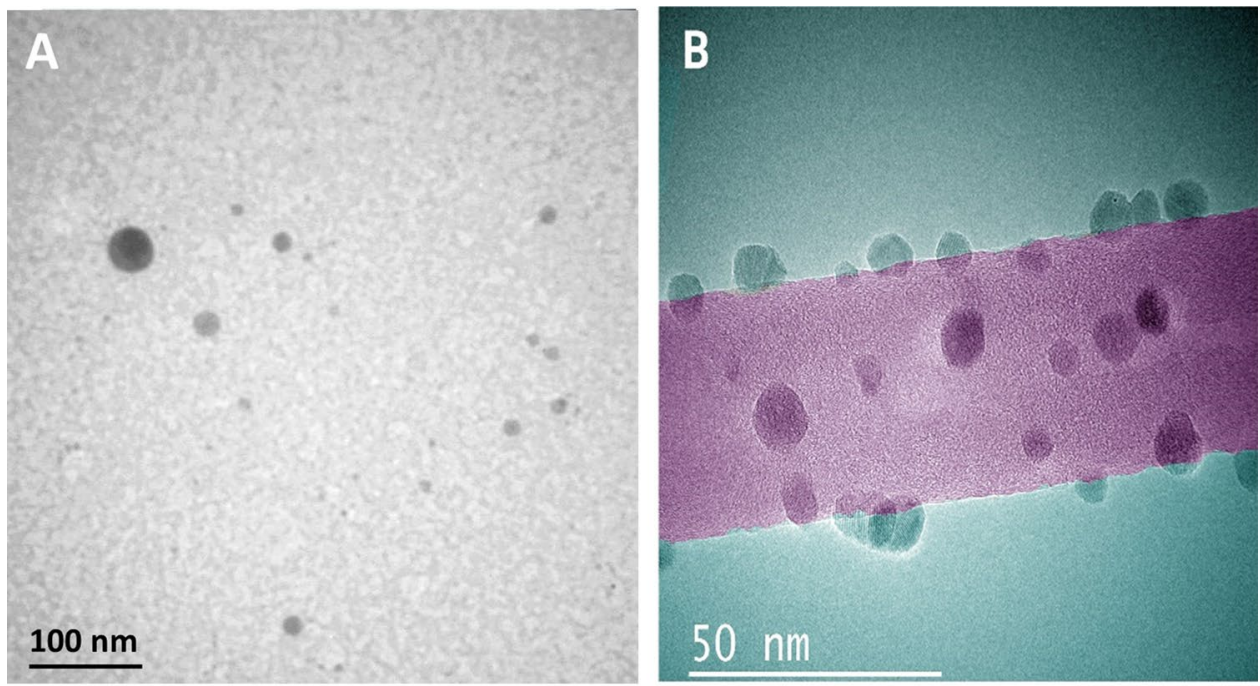